\title{
ALTERAÇÕES ORTOPÉDICAS NA AIDS
}

\author{
ORTHOPEDIC COMPLICATIONS IN HIV PATIENTS
}

Ana Lúcia Lei Munhoz Lima', Alexandre Leme Godoy', Priscila Rosalba Domingos Oliveira', Ricardo Gomes Gobbi', Camila de Almeida Silva', Patricia Bernardelli Martino', Eliana Bataggia Gutierrez², Maria Clara Gianna², Gilberto Luis Camanho'

\section{RESUMO}

O aumento considerável da expectativa de vida dos pacientes infectados pelo HIV na era do tratamento antirretroviral de alta potência, resulta em importantes alterações metabólicas e osteoarticulares decorrentes do prolongado tempo de infecção viral e desse tratamento. As complicações ortopédicas mais frequentes são as alterações da mineralização óssea, a osteonecrose, síndrome do túnel do carpo e capsulite adesiva glenoumeral, com padrão de apresentação clínica, evolução natural da doença e resposta terapêutica diferentes daqueles da população geral. Os relatos da literatura são iniciais e a experiência do serviço multidisciplinar do Instituto de Ortopedia e Traumatologia da USP permite avanço no conhecimento das diversas patologias envolvidas e o desenvolvimento de protocolos de tratamento adequados a esses diagnósticos.

Descritores - HIV; Ortopedia; Diagnóstico

\section{ABSTRACT}

The considerable increase of the life expectancy of HIV-infected patients in the age of highly-powerful antiretroviral treatment results in important metabolic and bone-joint changes resulting from a long-lasting viral infection time and from this treatment. The most common orthopaedic complications are bone mineralization changes, osteonecrosis, carpal tunnel syndrome and gleno-humeral adhesive capsulitis, with different clinical presentation features, natural disease progression and therapeutic response compared to the overall population. Literature reports are initial, and the experience of the multidisciplinary service of the University of São Paulo's Institute of Orthopaedics and Traumatology enables us a more in-depth knowledge about the various pathologies involved and the development of treatment protocols that are appropriate to these diagnoses.

Descritores - HIV; Orthopaedics; Diagnosis

\section{INTRODUÇÃO}

Com o aumento considerável da expectativa de vida dos pacientes infectados pelo HIV na era do tratamento antirretroviral (TARV) de alta potência, são observadas algumas consequências do prolongado tempo de infecção viral e desse tratamento. As consequências metabólicas ocorrendo nesse contexto são exploradas em várias publicações na literatura, principalmente a síndrome lipodistrófica. Atualmente, a observação crescente de alterações osteoarticulares nesses pacientes é objeto de estudo mais detalhado, no intuito da detecção de suas eventuais causas e abordagem terapêutica mais adequada.

Dentre as complexas alterações metabólicas da infecção crônica pelo HIV e seu tratamento, observa-se diminuição da mineralização óssea em grande percentagem dos doentes, resultante de vários fatores presentes no próprio hospedeiro, no vírus e nos antirretrovirais (ARV). O osso é constantemente remodelado pelo sincronismo entre sua formação e reabsorção, que pode ser desregulado durante a infecção pelo HIV. Quando a mineralização óssea diminui, a osteopenia ocorre, podendo resultar em osteoporose.

\footnotetext{
1. Instituto de Ortopedia e Traumatologia do Hospital das Clínicas da Faculdade de Medicina da Universidade de São Paulo (IOT).

2. Centro de Referência e Treinamento em DST/AIDS da Secretaria de Saúde do Estado de São Paulo.

3. Serviço de Extensão ao Atendimento de Pacientes HIV/Aids da Divisão de Moléstias Infecciosas e Parasitárias do Hospital das Clínicas da Universidade de São Paulo.
}

Trabalho realizado no Instituto de Ortopedia e Traumatologia do Hospital das Clínicas da Faculdade de Medicina da Universidade de São Paulo (IOT).

Correspondência: Rua Ovídio Pires de Campos, 333 - $3^{\circ}$ and., CEP 05403-010 - São Paulo, SP - Brasil. Tel.: (11) 3069-7812 - fax: (11) $3069-6888$. 
As alterações osteoarticulares mais frequentemente relatadas nos pacientes infectados pelo HIV por longo período e que utilizam TARV são a osteopenia/osteoporose, osteonecrose, síndrome do túnel do carpo e capsulite adesiva de ombros.

\section{Osteopenia/osteoporose}

Segundo a Organização Mundial de Saúde, as definições de osteopenia e osteoporose são baseadas em resultados observados na densitometria óssea ${ }^{(1)}$. A osteoporose é definida quando essa relação é inferior a 2 vezes o desvio padrão, e a osteopenia, quando o resultado se situa entre -1 e -2 vezes o desvio padrão ${ }^{(2)}$. A osteoporose pode ser considerada grave quando, além desse critério, o paciente apresenta fratura (Figura 1).

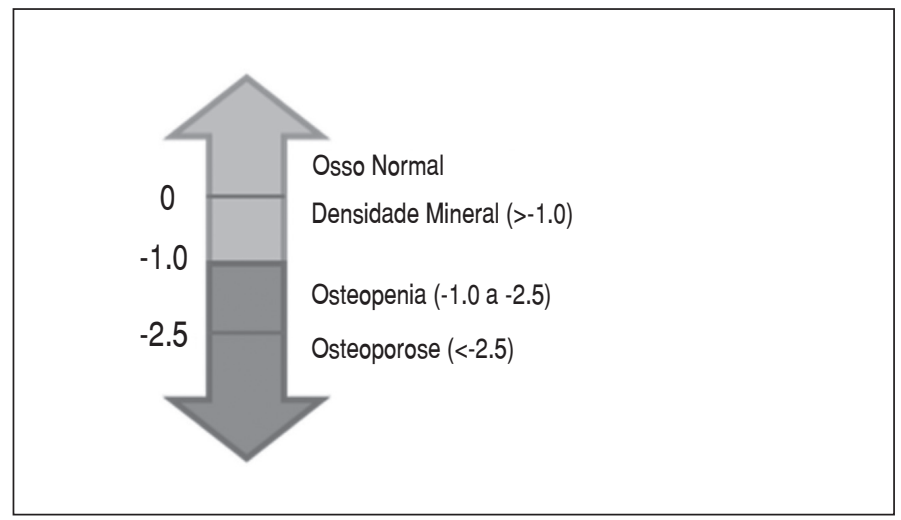

Figura 1 - Representação gráfica das faixas de normalidade e de alteração da densidade mineral óssea baseada em desvio padrão em relação à população geral

Diversos estudos têm demonstrado alta prevalência dessas alterações em pacientes infectados pelo HIV segundo esses critérios ${ }^{(1-4,6-11)}$. Múltiplos fatores são relacionados como causadores da osteopenia, dentre eles, efeitos diretos do vírus sobre as células osteogênicas; ativação persistente de citocinas pró-inflamatórias, principalmente $\mathrm{TNF} \alpha$ e interleucina-1; alterações no metabolismo da vitamina $\mathrm{D}$, com deficiência da 1,25 di-hidrovitamina D; e ainda, participação de anormalidades mitocondriais relacionadas com a acidemia lática e o desenvolvimento de doenças oportunistas ${ }^{(4,5)}$ (Figura 2).

Em relação à influência do TARV, existem estudos mostrando maior risco quando utilizados inibidores de protease (IP), sabendo-se que o indinavir inibe a formação óssea e o ritonavir inibe a diferenciação e função dos osteoclastos ${ }^{(1,6,7,9-11)}$. Com relação aos inibidores da

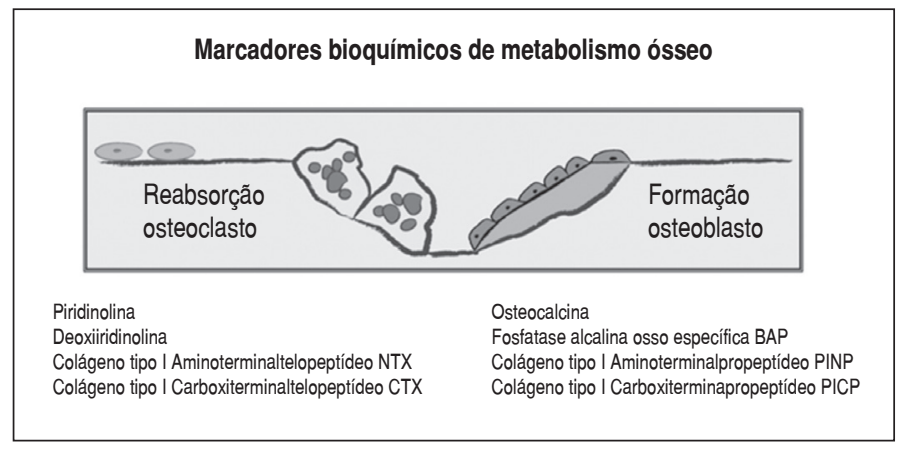

Figura 2 - Marcadores bioquímicos de metabolismo ósseo

transcriptase reversa, os relatos mais recentes têm ligado o tenofovir à ocorrência de osteomalácia e síndrome de Fanconi ${ }^{(12)}$. Ainda, outros fatores podem contribuir para acelerar a perda óssea, como: deficiências nutricionais, baixos níveis de cálcio sérico, imobilização, hipogonadismo, hipertireoidismo, hiperparatireoidismo, insuficiência renal, uso de opioides ou heroína, uso de corticosteroides, período pós-menopausa para mulheres e consumo de álcool superior a 16g/dia ${ }^{(1,8)}$ (Figuras 3 e 4)

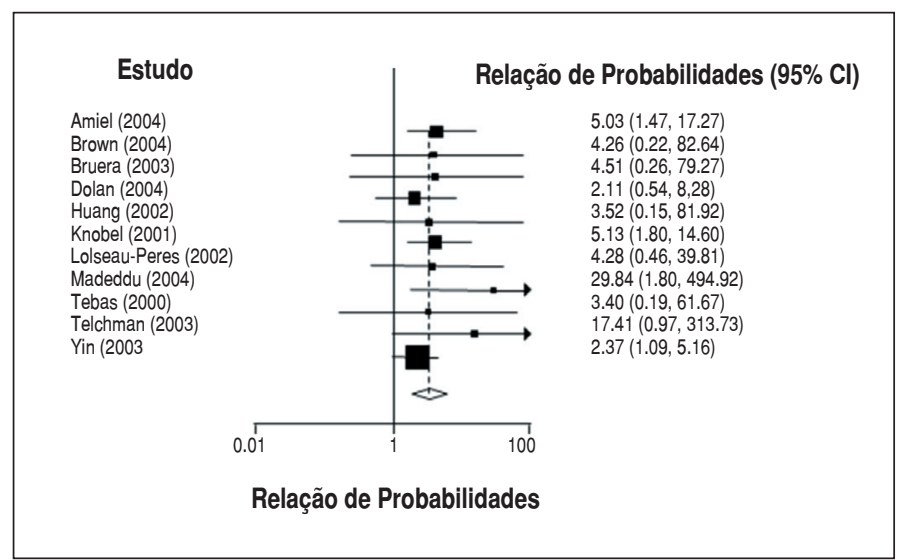

Figura 3 - Metanálise: risco de osteoporose em pacientes HIV e população controle

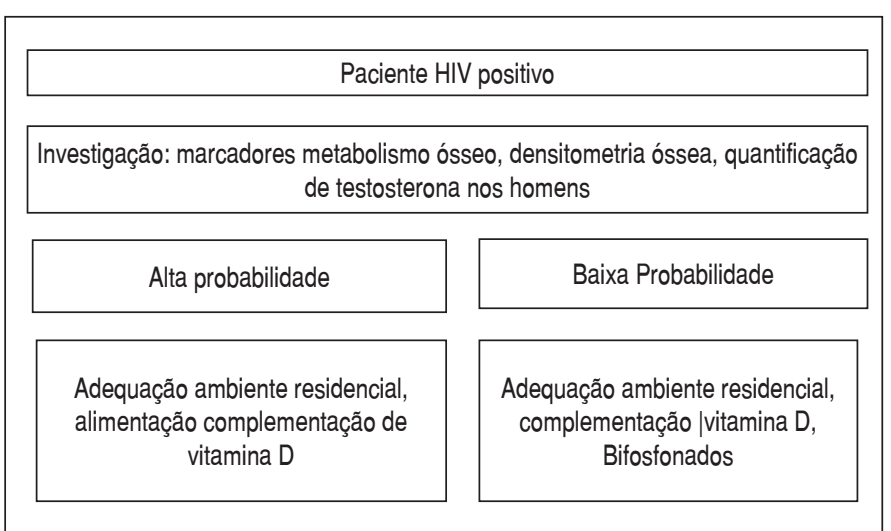

Figura 4 - Algoritmo: investigação e prevenção de complicação da diminuição de densidade mineral óssea 
Em relação ao tratamento da osteoporose, a principal medida é a prevenção, através do estímulo da atividade física e alimentação adequada nas primeiras três décadas de vida, para que se atinja a formação máxima de massa óssea.

A ingestão de cálcio e a administração suplementar de vitamina $\mathrm{D}$ devem fazer parte de qualquer regime terapêutico para a osteoporose. Na mulher pós-menopausa, a terapia de reposição hormonal é importante método de prevenção da osteoporose. Quanto à terapia medicamentosa, existem basicamente duas classes medicamentosas: os agentes antirreabsorção do tecido ósseo e agentes estimuladores da formação óssea.

\section{Osteonecrose}

A ocorrência de osteonecrose em pacientes com HIV tem sido relatada desde 1990, com incidências progressivamente crescentes e superiores às da população ge$\mathrm{ral}^{(7,8)}$. A incidência anual de osteonecrose sintomática na população geral é estimada entre 0,010 e $0,135 \%{ }^{(13)}$.

Estudos recentes que utilizaram ressonância magnética (RM) para detecção da osteonecrose em pacientes portadores do HIV estimaram a incidência dessa condição em aproximadamente 4\%. A incidência de bilateralidade varia de 35 a $80 \%{ }^{(13)}$.

$\mathrm{Na}$ população geral, são conhecidos alguns fatores de risco e condições associadas ao desenvolvimento da osteonecrose, como: uso de corticosteroides sistêmicos, alcoolismo, hiperlipidemia, anemia falciforme, coagulopatias, doença de Gaucher, lúpus eritematoso sistêmico, artrite reumatoide, hiperuricemia e gota, radioterapia, obesidade, pancreatite, sequela de fraturas, quimioterapia, vasculites e tabagismo. Além desses fatores, nos pacientes infectados pelo HIV, estão envolvidos no desenvolvimento da osteonecrose: a dislipidemia, o uso de acetato de megestrol e anabolizantes, a reposição de testosterona, bem como as vasculites que predispõem a trombose intraóssea pela presença de anticorpos anticardiolipina e pela deficiência da proteína S. Ainda, o próprio tratamento antirretroviral pode estar relacionado com o desenvolvimento crescente da osteonecrose ${ }^{(7,8,13)}$.

Para o diagnóstico da osteonecrose, devem ser observados sinais clínicos, como a presença de dor articular e limitação do grau de movimento. As articulações mais frequentemente envolvidas são quadris, uni ou bilateralmente, joelhos, tornozelos, cotovelos e ombros ${ }^{(14,15)}$.

Deve-se ressaltar que o intervalo entre as alterações radiológicas e os sintomas clínicos pode ser longo, variando de três a oito anos. A radiografia simples da arti- culação tem baixa sensibilidade diagnóstica no início da doença. São achados radiológicos frequentes indicadores de osteonecrose: esclerose cística, radioluzência subcondral, colapso ósseo e alterações degenerativas articulares. A tomografia computadorizada sem contraste acrescenta poucas informações à radiologia simples. A RM possui 99\% de sensibilidade e especificidade para o diagnóstico desde a fase inicial. A cintilografia óssea, apesar de pouco específica, pode ser utilizada no estadiamento e para a busca de focos ocultos assintomáticos ${ }^{(14,15)}$.

O tratamento varia com o estágio da doença. No paciente portador de HIV, é importante excluir ou controlar outros fatores de risco que não os da própria doença e medicação ARV. Em indivíduos oligossintomáticos, o tratamento pode ser baseado no uso de analgésicos e anti-inflamatórios não hormonais ${ }^{(6)}$.

Nos estágios iniciais podem ser utilizados procedimentos de descompressão da área com necrose, associada ou não a enxertos corticoesponjosos livres ou pediculados. Com o progredir da doença, quando se iniciam alterações da congruência articular, podem estar indicados procedimentos como osteotomias, hemiartroplastias ou artroplastias unicompartimentais, e, nos casos mais avançados, a solução é a artroplastia total ${ }^{(14)}$.

\section{Síndrome do túnel do carpo}

A incidência na população geral está em torno de 3,8\% ao exame clínico e, quando utilizada a eletroneuromiografia, 2,7\%. Na população HIV-positiva, a incidência manteve-se muito próxima daquela da população geral ${ }^{(16,17)}$.

Essa síndrome tem sido relacionada com o uso de TARV, especialmente com os IPs, e seria decorrente dos distúrbios metabólicos já conhecidos e depósito de material mixedematoso no túnel do carpo, com a consequente compressão nervosa. Outros fatores são associados ao desenvolvimento dessa síndrome nos pacientes portadores de HIV/AIDS, como atividades profissionais, hipotireoidismo, hiperglicemia, artrite reumatoide, obesidade e distúrbios metabólicos variados. Portanto, a correlação direta com a presença do HIV e o TARV ainda é questionável ${ }^{(16,17)}$.

O tratamento baseia-se no estadiamento da síndrome compressiva. Na fase leve, o tratamento é conservador, com o uso de talas noturnas e o emprego de medicações anti-inflamatórias ${ }^{(18)}$. Nas fases moderadas e graves está indicado o tratamento cirúrgico. Este pode ser feito de forma convencional ou por via endoscópica ${ }^{(18)}$. Em ambos os procedimentos realiza-se a descompressão do nervo mediano através da abertura do retináculo dos flexores. 


\section{Capsulite adesiva}

A capsulite adesiva tem sido associada aos pacientes portadores do HIV recebendo esquema ARV com $\mathrm{IPs}^{(19)}$. Os casos relatados na literatura limitam-se a envolvimento do ombro, sugerindo que outras localizações são $\operatorname{raras}^{(19,20)}$. Os sintomas característicos dessa afecção incluem dor progressiva uni ou bilateral nos ombros, com restrição ativa e passiva do arco de movimento. Classicamente, o inicio dos sintomas é insidioso, ocorrendo aproximadamente 12 a 14 meses após o inicio do uso dos IPs ${ }^{(19)}$. A radiografia simples pode demonstrar rarefação óssea ocasionada pelo desuso; entretanto, a artrorressonância magnética é o exame de eleição para o diagnóstico. Os sintomas tendem a regredir espontaneamente após um período de seis a 24 meses com a instituição do tratamento adequado e interrupção do TARV ${ }^{(19,20)}$.

$\mathrm{O}$ tratamento das capsulites adesivas depende de seu tempo de evolução e da gravidade das aderências. Nos casos mais leves, o tratamento conservador com medicamentos analgésicos, anti-inflamatórios e fisioterapia é o mais indicado ${ }^{(19,21)}$. Nos casos mais graves, que não respondem ao tratamento conservador, o tratamento artroscópico é o mais indicado, seguido de mobilização precoce. Temos evitado a indicação de manipulação isoladamente devido à maior incidência de fraturas do terço proximal do úmero e também ao pós-operatório mais doloroso, que dificulta a mobilização precoce ${ }^{(21)}$.

\section{DISCUSSÃO}

Considerando a prevalência e importância das alterações osteoarticulares, o IOT iniciou em março de 2006 o atendimento de pacientes HIV/AIDS com queixas ortopédicas, encaminhados de dois centros de referência em atendimento de pacientes infectados pelo HIV.

No período de março de 2006 a março de 2008, dos 206 pacientes avaliados, 83 foram matriculados no ambulatório, totalizando 614 atendimentos entre consultas iniciais e retornos.

Os pacientes analisados possuíam tempo prolongado de infecção pelo HIV, com média de 114 meses desde o diagnóstico. Também apresentavam exposição prolongada o TARV, com tempo médio de 96 meses de uso. Dentre os medicamentos mais utilizados encontramos lamivudina, zidovudina e nelfinavir. Havia histórico de uso de IPs em $72 \%$ da casuística (Figura 5).

No momento da avaliação, somente $8 \%$ dos pacientes apresentavam níveis de CD4 abaixo de 200 células/ $\mathrm{mm}^{3}$ e $74 \%$, carga viral indetectável.

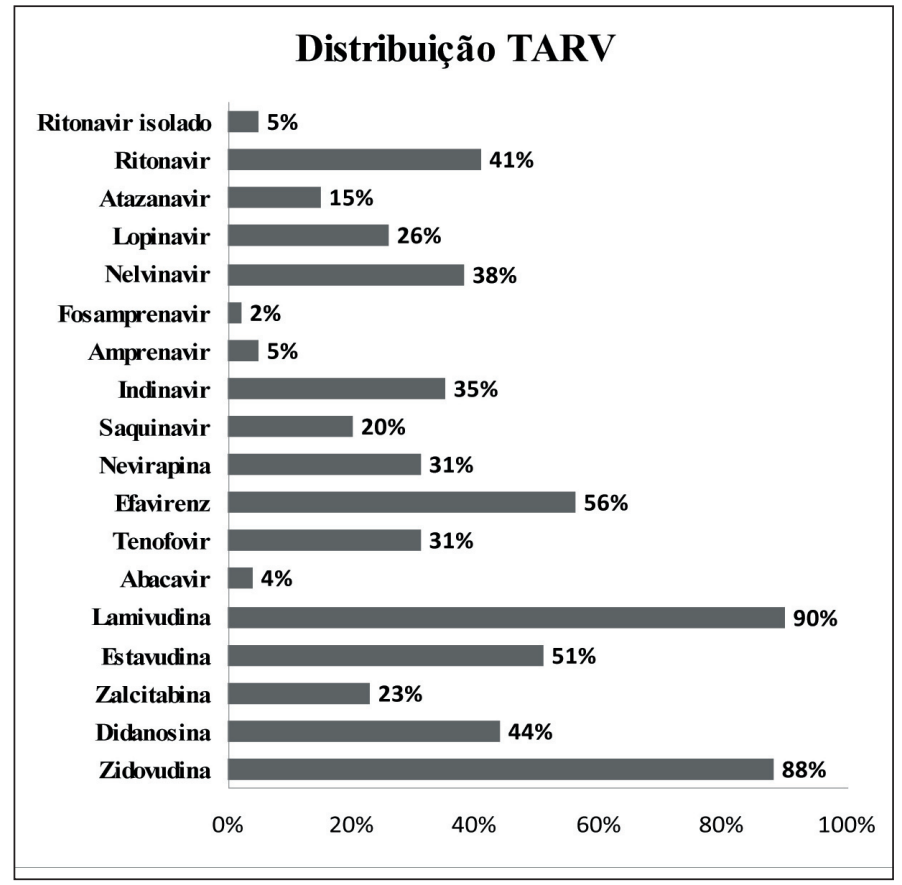

Figura 5 - Distribuição do TARV entre os pacientes infectados pelo HIV em acompanhamento no ambulatório do IOT

A alteração ortopédica mais prevalente na população estudada foi a osteonecrose, com incidência de $12 \%$. A articulação do quadril foi a mais acometida, com achado de bilateralidade em todos os casos. A bilateralidade de $100 \%$ é facilmente explicada por todos os casos serem de origem secundária, o que eleva esse índice (Figura 6 e Tabela 1).

A principal queixa dos pacientes consistiu inicialmente apenas em dor no quadril, com limitação do movimento e claudicação durante a evolução, acompanhando o quadro clínico clássico da doença.

\section{Distribuição por topografia das lesões ortopédicas}

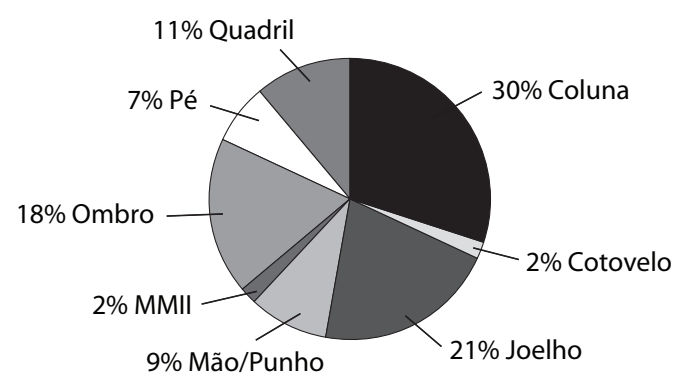

Figura 6 - Distribuição por topografia das lesões ortopédicas em pacientes infectados pelo HIV em acompanhamento no ambulatório do IOT 
Tabela 1 - Alterações osteoarticulares

\begin{tabular}{l|l|l|l}
\hline Membros superiores & Membros inferiores & Degenerativas & Esqueleto axial \\
\hline $\begin{array}{l}\text { Osteonecrose cabeça } \\
\text { úmero }\end{array}$ & $\begin{array}{l}\text { Osteonecrose cabeça } \\
\text { fêmur }\end{array}$ & Tendinopatias & Lombalgia mecânica \\
\hline Síndrome túnel carpo & $\begin{array}{l}\text { Osteonecrose } \\
\text { côndilos fêmur }\end{array}$ & Lesões musculares & $\begin{array}{l}\text { Colapso corpo } \\
\text { vertebral }\end{array}$ \\
\hline $\begin{array}{l}\text { Capsulite adesiva } \\
\text { ombro }\end{array}$ & Metatarsalgia & & \\
\hline & $\begin{array}{l}\text { Síndrome } \\
\text { femoropatelar }\end{array}$ & & \\
\hline
\end{tabular}

Todos os pacientes diagnosticados com osteonecrose encontravam-se em fase tardia de evolução.

Esse fato pode indicar tanto curso mais agressivo da doença quanto maior demora para o diagnóstico, pro- vavelmente relacionada com as abundantes manifestações clínicas dessa população e a pouca valorização de queixas secundárias. Além disso, observamos no estudo uma tendência de progressão clínica da doença mais acelerada, com quadro doloroso mais intenso e padrão de resposta terapêutica não cirúrgica menos favorável que na população geral.

\section{CONCLUSÃO}

As complicações osteoarticulares mostram prevalência significativa na população que convive com o HIV em uso de terapia antirretroviral de alta potência; com padrão de apresentação clínica, evolução natural da doença e resposta terapêutica diferentes daqueles da população geral.

\section{REFERÊNCIAS}

1. Amorosa V, Tebas P. Bone disease and HIV infection. Clin Infect Dis. 2006; 42(1):108-14.

2. Bruera D, Luna N, David DO, Bergoglio LM, Zamudio J. Decreased bone mineral density in HIV-infected patients is independent of antiretroviral therapy. AIDS. 2003;17(3):1917-23.

3. Delaunay C, Loiseau-Peres S, Benhamou CL. Osteopenia and human immunodeficiency virus. Joint Bone Spine. 2002;69(2):105-8.

4. Dolan SE, Huang JS, Killilea KM, Sullivan MP, Aliabadi N, Grinspoon S. Reduced bone density in HIV-infected women. AIDS. 2004:18(3):475-83.

5. Seminari E, Castagna A, Soldarini A, Galli L, Fusetti G. Dorigatti F, et al.. Osteoprotegerin and bone turnover markers in heavily pretreated HIV-infected patients. British HIV Assoc. 2005;6:145-50.

6. Jain RG, Furfine ES, Pedneault L, White AJ, Lenhard. Metabolic complications associated with antiretroviral therapy. Antiviral Res. 2001;51(3):151-77.

7. Mondy K, Tebas P. Emerging bone problems in patients infected with human immunodeficiency virus. Clin Infect Dis. 2003;36(Suppl 2):S101-5.

8. Jain RG, Lenhard JM. Select HIV protease inhibitors alter bone and fat metabolism ex vivo. J Biol Chem. 2002;277(22):19247-50.

9. Mora S, Sala N, Bricalli D, Zuin G, Chiumello G, Viganò A. Bone mineral loss through increased bone turnover in HIV-infected children treated with highly active antiretroviral therapy. AIDS. 2001;15(14):1823-9.

10. Mora S, Zamproni I, Beccio S, Bianchi R, Giacomet V, Viganò A. Longitudinal changes of bone mineral density and metabolism in antiretroviral-treated human immunodeficiency virus-infected children. J Clin Endocrinol Metab. 2004:89(1):24-8.

11. Tan BM, Nelson RP Jr, James-Yarish M, Emmnueal P, Schurman SJ. Bone metabolism in children with human immunodeficiency virus infection receiving

highly active anti-retroviral therapy including a protease inhibitor. J Pediatr. 2001;139(3):447-51.

12. Parsonage MJ, Wilkins EGL, Snowden N, Issa BG, Savage MW. The development of hypophosphataemic osteomalacia with myopathy in two patients with HIV infection receiving tenofovir therapy. HIV Med. 2005;6(5):341-6.

13. Mahoney CR, Glesby MJ, DiCarlo EF, Peterson MGE, Bostrom MP. Total hip arthroplasty in patients with human immunodeficiency virus infection. Acta Orthop. 2005;76(2):198-203.

14. Allison GT, Bostrom MP, Glesby MJ. Osteonecrosis in HIV disease: epidemiology, etiologies and clinical management. AIDS. 2003;17(1):1-9.

15. Allen SH, Moore AL, Tyrer MJ, Holloway BJ, Johnson MA. Osteonecrosis of the knee in a patient receiving antiretroviral therapy. Int J STD AIDS. 2002;13(11):792-4.

16. Sclar G. Carpal tunnel syndrome in HIV-1 patients: a metabolic consequence of protease inhibitor use? AIDS. 2000;14(3):336-8

17. Asensio O, Caso JAA, Rojas R. Carpal tunnel syndrome in HIV patients? AIDS. 2002;16(6):948-50

18. Canale ST. editor. Campbell's operative orthopaedics. 10th ed. Philadelphia: Mosby; 2003.

19. De Ponti A, Vigano MG, Taverna E, Sansone V. Adhesive capsulitis of the shoulder in human deficiency vírus-positive patients during highly active antiretroviral therapy. J Shoulder Elbow Surg. 2006;15(2):188-90.

20. Atalay A, Ozdemir O, Guven GS, Basgoze. O. HIV infection and shoulder pain: a challenging case. Rheumatol Int. 2006;26(7):680-2.

21. Warner JJP, Allen A, Marks PH, Wong P. Arthroscopic release for chronic refratory adhesive capsulitis of the shoulder. $\mathrm{J}$ Bone Joint Surg Am. 1996;78(12):1808-16 